\title{
Eficacia del cidofovir intralesional en el tratamiento de papilomatosis respiratoria recurrente juvenil: una revisión sistemática
}

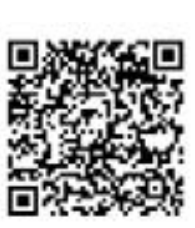

\author{
Efficacy of intralesional cidofovir for the treatment of juvenile \\ recurrent respiratory papillomatosis: a systematic review
}

\author{
Yuria Ablanedo-Terrazas, ${ }^{\star}$ Claudia Alvarado-de la Barrera, ${ }^{\ddagger}$ Daniel Bross-Soriano, ${ }^{*}$ \\ María del Carmen Cano-Salas, ${ }^{\S}$ Claudia Garrido-Galindo," Matilde Ruiz-Cruz, " José Schimelmitz-Idi*
}

\begin{abstract}
RESUMEN
Antecedentes: La papilomatosis respiratoria recurrente juvenil es una enfermedad crónica de etiología viral que habitualmente involucra numerosas cirugías para evitar la obstrucción respiratoria y mejorar la calidad de la voz. No existe cura para la enfermedad. Sin embargo, el cidofovir intralesional es el medicamento adyuvante más utilizado. Objetivo: Evaluar de manera sistemática la evidencia actual sobre la eficacia del cidofovir intralesional en el tratamiento de la papilomatosis respiratoria recurrente juvenil. Métodos: La búsqueda incluyó las bases de datos de Cochrane Library, PubMed, EMBASE, LILACS, SciELO, TripDatabase, National Guidelines Clearinghouse y ClinicalTrials.gov. Se incluyeron ensayos clínicos controlados aleatorizados y no aleatorizados, estudios de cohortes, estudios de casos y controles, series de casos y reportes de casos publicados hasta el 23 de febrero de 2019. Resultados: Se encontraron en total 91 referencias. De éstas, se eliminaron 69 tras leer los resúmenes en el primer escrutinio. Los 22 artículos restantes fueron evaluados con
\end{abstract}

\begin{abstract}
Background: Juvenile recurrent respiratory papillomatosis is a chronic disease of viral etiology that usually requires repeated surgical interventions in order to avoid respiratory obstruction an improve voice quality. There is no treatment to cure the disease. However, intralesional cidofovir is the most common adjuvant therapy. Objective: The objective of this study was to systematically review current evidence of the efficacy of intralesional cidofovir for the treatment of juvenile recurrent respiratory papillomatosis. Methods: The search strategy included the data bases of Cochrane Library, PubMed, EMBASE, LILACS, SciELO, TripDatabase, National Guidelines Clearinghouse and ClinicalTrials.gov. We included randomized and non-randomized controlled clinical trials, cohort studies, case-control studies, case series and case reports. Published studies until February 23, 2019 were considered. Results: We found a total of 91 references. Of these, 69 were eliminated in the first screening after reading the abstracts. The 22 remaining studies were assessed with STROBE and
\end{abstract}

* Práctica Médica Grupal en Otorrinolaringología, Centro Médico ABC. Ciudad de México, México.

‡ Dirección de Investigación, Instituto Nacional de Enfermedades Respiratorias Ismael Cosío Villegas. Ciudad de México, México.

$\S$ Dirección de Enseñanza, Instituto Nacional de Enfermedades Respiratorias Ismael Cosío Villegas. Ciudad de México, México.

" Servicio Clínico de Neumología Pediátrica, Instituto Nacional de Enfermedades Respiratorias Ismael Cosío Villegas. Ciudad de México, México.

ๆ Asociación para Evitar la Ceguera en México, Institución de Asistencia Privada. Ciudad de México, México.

\section{Recibido para publicación: 13/08/2019. Aceptado: 15/11/2019.}

Correspondencia: Yuria Ablanedo-Terrazas

Av. Carlos Graef Fernández Núm. 154, Col. Tlaxala,

Santa Fe, 05300, Cuajimalpa, Ciudad de México.

E-mail: yuria.ablanedo@gmail.com

\section{Abreviaturas:}

CONSORT = Consolidated Standards of Reporting Trials (por sus siglas en inglés).

GRADE = Grading of Recommendations, Assessment, Development and Evaluation (por sus siglas en inglés).

$\mathrm{PRR}=$ Papillomatosis respiratoria recurrente.

PRRA = Papillomatosis respiratoria recurrente del adulto.

PRRJ = Papillomatosis respiratoria recurrente juvenil.

STROBE = Strengthening the Reporting of Observational studies in Epidemiology (por sus siglas en inglés).

$\mathrm{VPH}=$ Virus del papiloma humano.

Este artículo puede ser consultado en versión completa en: www.medigraphic.com/analesmedicos 
STROBE y CONSORT. Sólo 13 estudios fueron seleccionados para la clasificación de la evidencia con GRADE. Debido al bajo nivel de calidad de la evidencia, todos los estudios fueron eliminados. Ningún artículo se incluyó para el análisis final. No fue posible efectuar un metaanálisis a partir de la literatura revisada. Conclusiones: La evidencia de la eficacia del cidofovir intralesional en el tratamiento de papilomatosis respiratoria recurrente juvenil es insuficiente. Es necesario efectuar ensayos clínicos, aleatorizados, controlados con placebo y doble ciego con un tamaño de muestra adecuado. Es probable que se requiera un estudio multicéntrico para reunir al número apropiado de pacientes.

Palabras clave: Papilomatosis respiratoria recurrente juvenil, cidofovir.

Nivel de evidencia: IIb

\section{INTRODUCCIÓN}

La papilomatosis respiratoria recurrente (PRR) es una enfermedad rara caracterizada por lesiones exofíticas de aspecto verrugoso, que tienden a reaparecer en las vías respiratorias superiores. ${ }^{1}$ Aunque la PRR es un neoplasma benigno que habitualmente involucra la laringe, su curso clínico es impredecible, dado que tiene el potencial para extenderse por el tracto respiratorio, y en raras ocasiones puede transformarse en neoplasia. ${ }^{2}$

El virus del papiloma humano (VPH) es el agente etiológico de la PRR. De los más de 100 tipos de VPH que se han identificado, el 6 y el 8 son los más comunes en la PRR. ${ }^{3}$ Se reconocen dos formas clínicas de PRR: la juvenil (PRRJ), que se manifiesta antes de los 13 años y la forma adulta (PRRA). ${ }^{4}$ La PRRJ suele tener un curso clínico más agresivo. El diagnóstico antes de los tres años de edad se asocia con una probabilidad 3.6 veces mayor de requerir más de cuatro procedimientos quirúrgicos por año y con una probabilidad casi dos veces mayor de tener dos o más sitios anatómicos afectados. Se estima que en Estados Unidos la incidencia de PRRJ es de 4.3 por 100,000 niños y la de PRRA es de 1.8 por 100,000 adultos. ${ }^{5,6}$

Actualmente no hay un tratamiento que logre curar la PRR, por lo que se requieren procedimientos quirúrgicos repetidos para evitar la obstrucción respiratoria y mejorar la calidad de la voz. ${ }^{7}$ Aun con el uso de técnicas quirúrgicas sofisticadas, las secuelas laríngeas son complicaciones frecuentes. Los niños que se han sometido a múltiples procedimientos quirúrgicos suelen presentar sinequias en la comisura anterior y estenosis glótica. ${ }^{8}$ A fin de reducir la progresión de la enfermedad y limitar el número de pro-
CONSORT. Only 13 studies were selected for the classification of evidence with GRADE. Because evidence was of low-quality, all studies were eliminated. No studies were included in the final analysis. It was not possible to perform a meta-analysis from this systematic review. Conclusions: We did not find high-quality evidence of the efficacy of intralesional cidofovir for the treatment of juvenile recurrent respiratory papillomatosis. Large, double-blind, placebo controlled, randomized trials are required to support the efficacy of cidofovir for the treatment of juvenile recurrent respiratory papillomatosis. It is likely that a multi-center trial will be required to enroll adequate patient numbers.

Keywords: Juvenile recurrent respiratory papillomatosis, cidofovir.

Level of evidence: $I I b$

cedimientos quirúrgicos, el uso de terapias adyuvantes está indicado en pacientes que requieren más de cuatro procedimientos quirúrgicos por año, con rápido crecimiento de papilomas que comprometen la vía aérea y con propagación distal de la enfermedad. ${ }^{9} \mathrm{El}$ cidofovir, un análogo de citocina que inhibe la replicación del DNA viral, frecuentemente se usa como medicamento adyuvante en el tratamiento de PRR. Aunque solamente está aprobado por la FDA para el tratamiento de retinitis por citomegalovirus, ${ }^{10}$ se ha utilizado fuera de esta indicación para el tratamiento de PRR debido a su efecto supresor sobre el VPH. ${ }^{11}$ La administración intralesional del cidofovir tiene una tolerancia aceptable y una toxicidad sistémica limitada, ${ }^{12}$ además de que en diversos estudios se han observado resultados prometedores de su uso como terapia adyuvante. ${ }^{13}$

El objetivo es revisar de manera sistemática la evidencia actual sobre la eficacia del cidofovir intralesional en el tratamiento de la PRRJ.

\section{MÉTODOS}

\section{Estrategia de búsqueda}

Se identificaron los artículos originales publicados sobre el uso de cidofovir en el tratamiento de PRRJ mediante la búsqueda en Cochrane Library, PubMed, EMBASE, LILACS, SciELO, TripDatabase, National Guidelines Clearinghouse y ClinicalTrials. gov. Se diseñaron estrategias de búsqueda combinando los diferentes términos $\mathrm{MeSH}$ con el siguiente algoritmo: («laryngeal papillomatosis» $[\mathrm{MeSH}$ Terms] o «respiratory papillomatosis»[All Fields]) y («cidofovir»[MeSH Terms] o «vistide»[All Fields]). 
Se usó la misma estrategia de búsqueda en todas las bases de datos mencionadas. Se incluyó la literatura publicada hasta el 23 de febrero de 2019.

\section{Criterios de inclusión}

En la búsqueda original, se consideraron los ensayos clínicos controlados aleatorizados y no aleatorizados, estudios de cohortes, estudios de casos y controles, series de casos y reportes de casos. Solamente se incluyeron los estudios en humanos, con pacientes de 0 a 18 años de edad, con diagnóstico de papilomatosis respiratoria recurrente juvenil y tratados con cidofovir intralesional.

\section{Recolección de datos y análisis}

Se evaluaron únicamente los estudios en inglés y español. No hubo restricción en cuanto al año de publicación. Los estudios fueron evaluados de forma independiente por dos autores (José Schimelmitz-Idi y Yuria Ablanedo-Terrazas), y cualquier desacuerdo se resolvió mediante la discusión con un tercer autor (Daniel Bross-Soriano). El desenlace de interés fue la disminución en el número de recurrencias de la enfermedad.

\section{Evaluación de calidad de la evidencia de los artículos incluidos}

En un primer análisis se evaluó el ensayo clínico con la herramienta CONSORT. ${ }^{14}$ De igual forma, los estudios de cohorte y las series de casos se evaluaron con la herramienta STROBE para el reporte de estudios observacionales. ${ }^{15}$ Los artículos seleccionados en esta primera fase se sometieron a un segundo análisis en el que se categorizó la evidencia presentada en los estudios de acuerdo al sistema GRADE. ${ }^{16}$ Los autores revisaron de manera independiente las limitaciones en el diseño y ejecución de los estudios (riesgos de sesgo), la inconsistencia en los resultados, la incertidumbre acerca de que la evidencia sea directa, la imprecisión, el sesgo de publicación y el reporte de otras posibles amenazas a la validez.

\section{RESULTADOS}

Con la búsqueda realizada se encontraron en total 91 referencias. De éstas, se eliminaron 69 tras leer los resúmenes en el primer escrutinio (39 se eliminaron por referirse a temas no relacionados con el desenlace de interés, 12 se eliminaron por ser revisiones bibliográficas, 8 se eliminaron por encon- trarse en un idioma distinto a inglés o español, 7 por ser estudios realizados en adultos y 3 por ser editoriales). De esta manera, quedaron 22 artículos relacionados específicamente con el uso de cidofovir en papilomatosis respiratoria recurrente juvenil, enfocados a la recurrencia de la enfermedad. Este grupo de estudios incluyó un ensayo clínico aleatorizado, ${ }^{17} 18$ series de casos, ${ }^{18-35}$ un estudio de casos y controles ${ }^{36}$ y dos reportes de casos. ${ }^{37,38}$ El ensayo clínico aleatorizado se eliminó al analizarse con la herramienta CONSORT. Al evaluar los estudios observacionales con la herramienta STROBE, solamente se aceptaron 13. El acuerdo entre los dos revisores en este escrutinio fue de $71.9 \%(\kappa=0.43)$. La calidad de la evidencia de los 13 artículos restantes se categorizó con la herramienta GRADE (Tabla 1). Tras recibir un nivel de calidad $a$ priori de acuerdo al diseño del estudio, todos los estudios fueron eliminados por factores tales como el riesgo de sesgo, inconsistencia, imprecisión o sesgo de publicación (Figura 1). Por lo anterior, no se incluyó ningún estudio para el análisis final. No fue posible efectuar un metaanálisis.

\section{DISCUSIÓN}

El objetivo de este estudio fue evaluar de manera sistemática la evidencia actual sobre la eficacia del cidofovir intralesional en el tratamiento de PRRJ. En la literatura revisada no encontramos estudios que cumplieran con los estándares básicos de calidad metodológica, por lo que consideramos que la evidencia de la eficacia del cidofovir intralesional en el tratamiento de PRRJ es insuficiente. Por lo anterior, no fue posible emitir una recomendación ni realizar un metaanálisis a partir de la evidencia proporcionada por los artículos revisados.

Hasta ahora, el uso de cidofovir intralesional en el tratamiento de PRRJ se ha basado en resultados prometedores derivados de reportes de casos, series de casos, un estudio de casos y controles y un ensayo clínico. No existen ensayos clínicos controlados con placebo, aleatorizados, dobles ciegos, bien diseñados, bien ejecutados, con un tamaño de muestra adecuado, que permitan sustentar la administración de cidofovir intralesional para el tratamiento de PRRJ. El único ensayo clínico fue publicado por McMurray en 2008. ${ }^{17}$ Este estudio tuvo un poder limitado, debido al tamaño de muestra insuficiente y al cambio de dosis de cidofovir durante la conducción del ensayo de 0.3 a $5 \mathrm{mg} / \mathrm{mL}$ en niños y de 0.75 a 5 $\mathrm{mg} / \mathrm{mL}$ en adultos. Los autores encontraron mejoría 
en la escala de severidad de Derkay a los 12 meses postratamiento, sin embargo, este mismo efecto también se observó en el grupo placebo, por lo que no pudieron comprobar la eficacia del cidofovir en el tratamiento de la papilomatosis. Las series de casos no cuentan con un grupo control, y en el estudio de la PRRJ eso es particularmente grave puesto que la enfermedad tiende a la mejoría. Es decir, solamente mediante la inclusión de un grupo placebo, se puede definir si el efecto positivo se debe al tratamiento o es simplemente reflejo de la historia natural de la enfermedad. En cuanto al estudio de casos y controles, ${ }^{36}$ cabe mencionar que su validez se vio limitada por la falta de aleatorización, de cegamiento y de un grupo placebo, así como también por un tamaño de muestra insuficiente.

El estudio de enfermedades raras como la PRR presupone ciertas dificultades para analizar de manera conjunta los resultados de los diferentes estudios. Por ejemplo, existen variaciones importantes en cuanto a la concentración de cidofovir, el volumen, la dosis total, el número de administraciones, el intervalo entre los tratamientos y el tiempo de seguimiento. ${ }^{39}$ Otras variables importantes entre los estudios son los desenlaces de interés analizados, que pueden incluir la tasa de remisión, la recurrencia de la enfer-

Tabla 1: Características de los estudios analizados con la herramienta GRADE.

\begin{tabular}{|c|c|c|c|c|c|}
\hline Autor & Diseño del estudio & Número de pacientes & Desenlace de interés & Tiempo de seguimiento & $\begin{array}{l}\text { Régimen de dosificación del } \\
\text { cidofovir intralesional }\end{array}$ \\
\hline Ablanedo-Terrazas ${ }^{18}$ & Serie de casos & 9 niños & Tasa de cirugía & $19.2-40$ meses & $\begin{array}{l}\text { Concentración } 5 \mathrm{mg} / \mathrm{mL} \\
5 \text { intervenciones } \\
\text { con intervalo de } 1 \text { mes }\end{array}$ \\
\hline Bielecki²1 & Serie de casos & 10 niños & $\begin{array}{c}\text { Tasa de cirugía/escala } \\
\text { de Derkay }\end{array}$ & $\begin{array}{c}6 \text { meses (en algunos } \\
\text { pacientes) }\end{array}$ & $\begin{array}{c}\text { Concentración } 5-10 \mathrm{mg} / \mathrm{mL} \\
\text { 6-14 intervenciones } \\
\text { con intervalo de } 1 \mathrm{mes}\end{array}$ \\
\hline Naiman $^{24}$ & Serie de casos & 16 niños & $\begin{array}{c}\text { Tasa de cirugía/escala } \\
\text { de Derkay }\end{array}$ & $12-76$ meses & $\begin{array}{l}\text { Media de } 8.9 \text { inyecciones, } \\
\text { cada } 4 \text { semanas } \\
\text { Concentración } 5-7.5 \mathrm{mg} / \mathrm{mL}\end{array}$ \\
\hline Chung $^{25}$ & Serie de casos & 11 niños & $\begin{array}{c}\text { Tasa de cirugía/escala } \\
\text { de Derkay }\end{array}$ & $\begin{array}{l}10-45 \text { meses (media } \\
30.2 \text { meses) }\end{array}$ & $\begin{array}{c}4 \text { intervenciones } \\
\text { con intervalo de } 1 \mathrm{mes} \\
\text { Concentración } 5-10 \mathrm{mg} / \mathrm{mL}\end{array}$ \\
\hline $\mathrm{Lee}^{27}$ & Serie de casos & 16 pacientes (3 niños) & $\begin{array}{c}\text { Tasa de cirugía/escala } \\
\text { de Derkay }\end{array}$ & $\begin{array}{c}13-48 \text { meses (media } \\
25.4)\end{array}$ & $\begin{array}{l}\text { 1-9 inyecciones cada } 3 \text { semanas } \\
\text { Concentración } 2.5-5 \mathrm{mg} / \mathrm{mL}\end{array}$ \\
\hline Shirley $^{28}$ & Serie de casos & 11 niños & $\begin{array}{c}\text { Tasa de cirugía/escala } \\
\text { de Derkay }\end{array}$ & $\begin{array}{c}\text { Más de } 2 \text { años (media } \\
\text { de } 2.5 \text { años) }\end{array}$ & $\begin{array}{c}\text { Concentración } 5-7.5 \mathrm{mg} / \mathrm{mL} \\
4 \text { intervenciones } \\
\text { con intervalo de } 2 \text { semanas, } \\
\text { después a criterio del cirujano }\end{array}$ \\
\hline Naiman $^{29}$ & Serie de casos & 26 pacientes (10 niños) & Escala de Derkay & $\begin{array}{c}1-32 \text { meses (media } 9 \\
\text { meses) }\end{array}$ & $\begin{array}{c}\text { 1-7 inyecciones cada mes } \\
\text { (las primeras } 3 \text { ) } \\
\text { Concentración } 5 \mathrm{mg} / \mathrm{mL}\end{array}$ \\
\hline Chhetri30 & Serie de casos & 5 niños & Escala de Derkay & Media 66 semanas & $\begin{array}{c}\text { 8-22 inyecciones cada mes (las } \\
\text { primeras } 4 \text { ) } \\
\text { Concentración } 1 \mathrm{mg} / \mathrm{mL}\end{array}$ \\
\hline Pransky ${ }^{31}$ & Serie de casos & 11 niños & $\begin{array}{c}\text { Tasa de cirugía/escala } \\
\text { de Derkay }\end{array}$ & $\begin{array}{l}\text { 39-59 semanas (media } \\
51.6)\end{array}$ & 4-13 inyecciones cada 2-4 semanas \\
\hline $\mathrm{Akst}^{32}$ & Serie de casos & 11 niños & Escala de Derkay & 3-19 meses (media 9.3) & $\begin{array}{c}\text { 4-8 intervenciones } \\
\text { con intervalo de } 1 \mathrm{mes} \\
\text { Concentración } 5-10 \mathrm{mg} / \mathrm{mL}\end{array}$ \\
\hline Milczuk ${ }^{33}$ & Serie de casos & 4 niños & & & $\begin{array}{c}6 \text { inyecciones cada } 6-8 \text { semanas } \\
\text { Concentración } 5 \mathrm{mg} / \mathrm{mL}\end{array}$ \\
\hline Pransky ${ }^{35}$ & Serie de casos & 10 niños & Escala de Derkay & & $\begin{array}{l}\text { 8-18 inyecciones cada } 2 \text { semanas } \\
\text { Concentración } 5 \mathrm{mg} / \mathrm{mL}\end{array}$ \\
\hline Mandell ${ }^{36}$ & Casos y controles & $\begin{array}{c}7 \text { niños (4 en grupo } \\
\text { de cidofovir y } 3 \text { grupo } \\
\text { control) }\end{array}$ & Escala de Derkay & $16-56$ meses & $\begin{array}{l}\text { 2-7 intervenciones cada } 2 \text { meses } \\
\text { Concentración } 5 \mathrm{mg} / \mathrm{mL}\end{array}$ \\
\hline
\end{tabular}




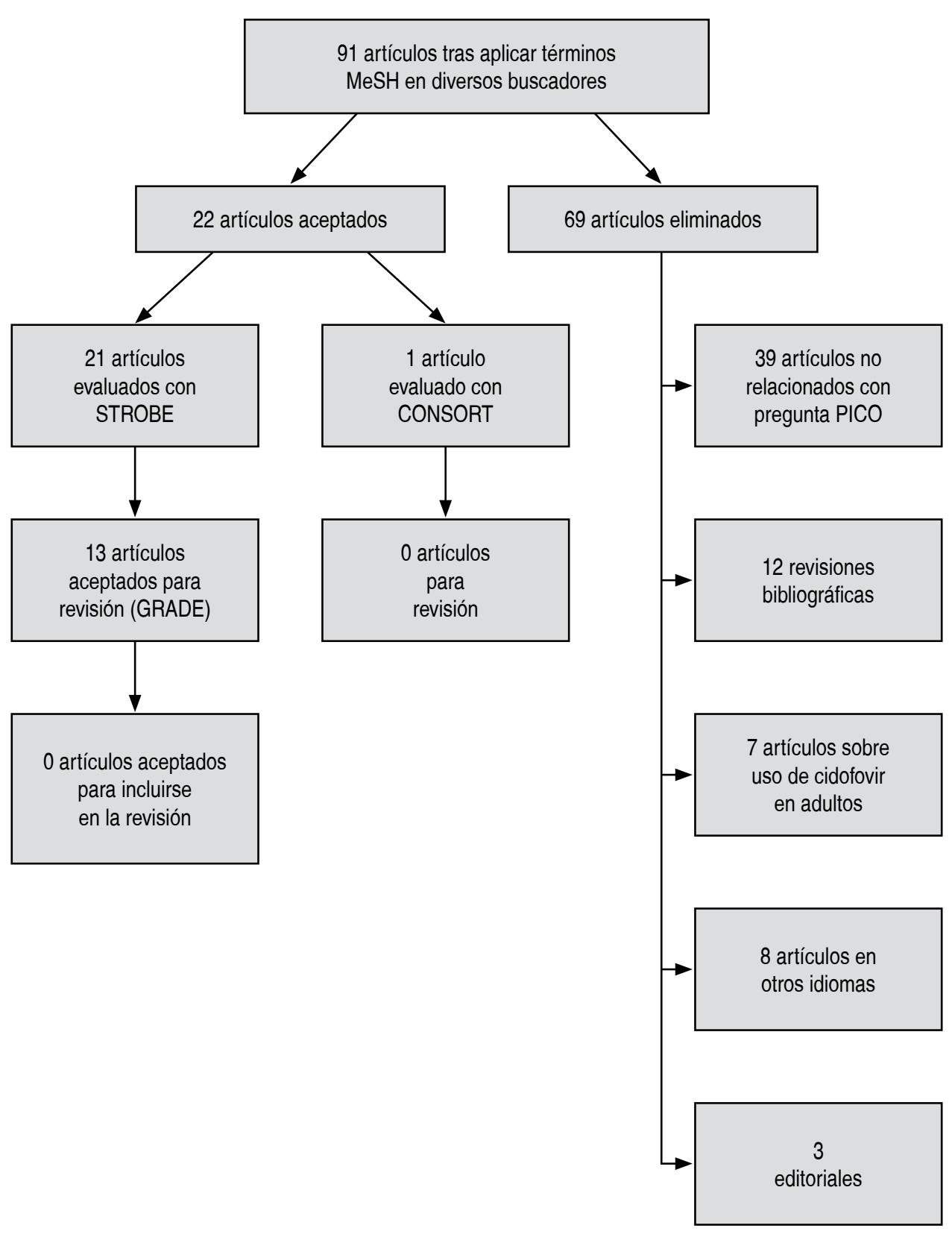

Figura 1:

Diagrama de flujo del estudio. medad, el intervalo entre cirugías, el puntaje en la escala Derkay, entre otros. ${ }^{40}$

La principal limitación de esta revisión sistemática es el nivel de evidencia de los estudios revisados. Es decir, la calidad y la fuerza de las recomendaciones en una revisión son tan sólidas como la calidad de los estudios revisados, ${ }^{16}$ que en este caso, sólo fueron series de casos, un estudio de casos y controles y un ensayo clínico. Otra limitación de esta revisión es que solamente se incluyeron los estudios en inglés y en español, lo cual representa un sesgo de idioma. Una limitación adicional es que no se incluyó la literatura gris, lo cual representa un sesgo de publicación hacia la inclusión de resultados positivos. Durante la búsqueda de artículos para esta revisión encontramos que se han publicado tres revisiones sistemáticas sobre el uso de cidofovir intralesional en PRR. Esto no necesariamente representa una limitación de nuestro estudio, puesto que estas revisiones se publicaron en $2007,{ }^{41} 2011^{42}$ y $2017,{ }^{40}$ en tanto que nuestro estudio abarca las publicaciones hasta 2019. Además, los objetivos de búsqueda fueron diferentes. 
Chadha y colaboradores ${ }^{41}$ hicieron una revisión sistemática de los agentes antivirales utilizados hasta el año 2007, que incluyeron aciclovir, ribavirina, cidofovir intravenoso e intralesional. Aunque en dicha revisión se concluyó que el cidofovir intralesional podría ser eficaz para el tratamiento de PRR, se recomendó la conducción de ensayos clínicos aleatorizados doble ciego para confirmar esto. En 2011, el mismo autor publicó una revisión sistemática sobre la eficacia y la seguridad del cidofovir intralesional en niños y adultos con PRRJ. ${ }^{42}$ En ese estudio se concluyó que la evidencia de la eficacia del cidofovir en el tratamiento de PRR era muy limitada, por lo que se recomendó que la terapia adyuvante en niños sólo se utilizara en casos excepcionales, pues podría tener menor eficacia y más riesgos potenciales a largo plazo que en adultos. En la revisión de Drejet publicada en 2017 sobre el uso de cidofovir intralesional y bevacizumab en pacientes con PRR del adulto, se concluyó que los estudios revisados tenían una calidad metodológica limitada. ${ }^{40}$

\section{CONCLUSIONES}

La evidencia de la eficacia del cidofovir intralesional en el tratamiento de PRRJ es insuficiente. Es necesario efectuar ensayos clínicos bien diseñados, aleatorizados, controlados con placebo, doble ciego y con tamaño de muestra adecuado. Es probable que se requiera un estudio multicéntrico para lograr incluir a un número adecuado de pacientes.

\section{AGRADECIMIENTO}

Agradecemos a la Asociación Médica del Centro Médico $\mathrm{ABC}$ por su invaluable apoyo en la obtención de diversos artículos utilizados en esta revisión.

\section{BIBLIOGRAFÍA}

1. Larson DA, Derkay CS. Epidemiology of recurrent respiratory papillomatosis. APMIS. 2010; 118: 450-454.

2. Steinberg BM, DiLorenzo TP. A possible role for human papillomaviruses in head and neck cancer. Cancer Metastasis Rev. 1996; 15: 91-112.

3. Dickens P, Srivastava G, Loke SL, Larkin S. Human papillomavirus 6, 11, and 16 in laryngeal papillomas. J Pathol. 1991; 165: 243-246.

4. Xue $\mathrm{Q}$, Wang $\mathrm{H}$, Wang J. Recurrent respiratory papillomatosis: an overview. Eur J Clin Microbiol Infect Dis. 2010; 29 (9): 1051-1054.

5. Derkay CS. Task force on recurrent respiratory papillomas. A preliminary report. Arch Otolaryngol Head Neck Surg. 1995; 121 (12): 1386-1391.

6. Armstrong LR, Derkay CS, Reeves WC. Initial results from the national registry for juvenile-onset recurrent respiratory papillomatosis. RRP Task Force. Arch Otolaryngol Head Neck Surg. 1999; 125: 743-748.

7. Omland T, Akre H, Lie KA, Jebsen P, Sandvik L, Brøndbo $\mathrm{K}$. Risk factors for aggressive recurrent respiratory papillomatosis in adults and juveniles. PLoS One. 2014; 9 (11): e113584.

8. Hermann JS, Pontes P, Weckx LL, Fujita R, Avelino M, Pignatari SS. Laryngeal sequelae of recurrent respiratory papillomatosis surgery in children. Rev Assoc Med Bras (1992). 2012; 58 (2): 204-208.

9. Katsenos S, Becker HD. Recurrent respiratory papillomatosis: a rare chronic disease, difficult to treat, with potential to lung cancer transformation: apropos of two cases and a brief literature review. Case Rep Oncol. 2011; 4: 162-171.

10. FDA Approved Drug Products. Vistide. [Consultado 8/ abril/2019]. Disponible en: https://www.accessdata. fda.gov/scripts/cder/daf/index.cfm?event=overview. process\&ApplNo $=020638$.

11. Tjon Pian Gi RE, Dietz A, Djukic V, Eckel HE, Friedrich $\mathrm{G}$, Golusinski W et al. Treatment of recurrent respiratory papillomatosis and adverse reactions following off-label use of cidofovir (Vistide®). Eur Arch Otorhinolaryngol. 2012; 269 (2): 361-362.

12. Broekema FI, Dikkers FG. Side-effects of cidofovir in the treatment of recurrent respiratory papillomatosis. Eur Arch Otorhinolaryngol. 2008; 265: 871-879.

13. Carifi M, Napolitano D, Morandi M, Dall'Olio D. Recurrent respiratory papillomatosis: current and future perspectives. Ther Clin Risk Manag. 2015; 11:731-38.

14. Schulz KF, Altman DG, Moher D, the CONSORT Group. CONSORT 2010 Statement: updated guidelines for reporting parallel group randomised trials. BMJ. 2010; 340: 698-702.

15. von Elm E, Altman DG, Egger M, Pocock SJ, Gøtzsche PC, Vandenbroucke JP; STROBE Initiative. The Strengthening the Reporting of Observational Studies in Epidemiology (STROBE) statement: guidelines for reporting observational studies. J Clin Epidemiol. 2008; 61: 344-349.

16. Guyatt GH, Oxman AD, Vist GE, Kunz R, Falck-Ytter Y, Alonso-Coello P et al. GRADE: an emerging consensus on rating quality of evidence and strength of recommendations. BMJ. 2008; 336: 924-926.

17. McMurray JS, Connor N, Ford CN. Cidofovir efficacy in recurrent respiratory papillomatosis: a randomized, doubleblind, placebo-controlled study. Ann Otol Rhinol Laryngol. 2008; 117: 477-483.

18. Ablanedo-Terrazas Y, Soda-Merhy A, Hernández-Palestina M, Ormsby CE, Reyes-Terán G. Intralesional cidofovir in severe juvenile respiratory papillomatosis. B-ENT. 2012; 83: 197-202.

19. Graupp M, Gugatschka M, Kiesler K, Reckenzaun E, Hammer G, Friedrich G. Experience of 11 years use of cidofovir in recurrent respiratory papillomatosis. Eur Arch Otorhinolaryngol. 2013; 270: 641-646.

20. Valera F, Maldonato L, Lima J, Küpper DS, Lacerda RN, Mamede R et al. Efficacy of cidofovir in recurrent juvenile respiratory papillomatosis. Braz J Otorhinolaryngol. 2010; 76: 713-717.

21. Bielecki I, Mniszek J, Cofała M. Intralesional injection of cidofovir for recurrent respiratory papillomatosis in children. Int J Pediatr Otorhinolaryngol. 2009; 73: 681-684.

22. Shi ZP, Wang CH, Lee JC, Lin YS. Cidofovir injection for recurrent laryngeal papillomatosis. J Chin Med Assoc. 2008; 71: 143-146.

23. Pudszuhn A, Welzel C, Bloching M, Neumann K. Intralesional cidofovir application in recurrent laryngeal papillomatosis. Eur Arch Otorhinolaryngol. 2007; 264: 63-70. 
24. Naiman AN, Ayari S, Nicollas R, Landry G, Colombeau B, Froehlich P. Intermediate-term and long-term results after treatment by cidofovir and excision in juvenile laryngeal papillomatosis. Ann Otol Rhinol Laryngol. 2006; 115: 667672.

25. Chung BJ, Akst LM, Koltai PJ. 3.5-year follow-up of intralesional cidofovir protocol for pediatric recurrent respiratory papillomatosis. Int J Pediatr Otorhinolaryngol. 2006; 70: 1911-1917.

26. Sheahan P, Sexton S, Russell JD. Is intralesional cidofovir worthwhile in juvenile recurrent respiratory papillomatosis? J Laryngol Otol. 2006; 120: 561-565.

27. Lee AS, Rosen CA. Efficacy of cidofovir injection for the treatment of recurrent respiratory papillomatosis. J Voice. 2004; 18: 551-556.

28. Peyton-Shirley W, Wiatrak B. Is cidofovir a useful adjunctive therapy for recurrent respiratory papillomatosis in children? Int J Pediatr Otorhinolaryngol. 2004; 68: 413-418.

29. Naiman AN, Ceruse P, Coulombeau B, Froehlich P. Intralesional cidofovir and surgical excision for laryngeal papillomatosis. Laryngoscope. 2003; 113: 2174-2181.

30. Chhetri DK, Shapiro NL. A scheduled protocol for the treatment of juvenile recurrent respiratory papillomatosis with intralesional cidofovir. Arch Otolaryngol Head Neck Surg. 2003; 129: 1081-1085.

31. Pransky SM, Albright JT, Magit AE. Long-term follow-up of pediatric recurrent respiratory papillomatosis managed with intralesional cidofovir. Laryngoscope. 2003; 113: 1583-1587.

32. Akst LM, Lee W, Discolo C, Knott D, Younes A, Koltai PJ. Stepped-dose protocol of cidofovir therapy in recurrent respiratory papillomatosis in children. Arch Otolaryngol Head Neck Surg. 2003; 129: 841-846.

33. Milczuk HA. Intralesional cidofovir for the treatment of severe juvenile recurrent respiratory papillomatosis: longterm results in 4 children. Otolaryngol Head Neck Surg. 2003; 128: 788-794.
34. El Hakim H, Waddell AN, Crysdale WS. Observations on the early results of treatment of recurrent respiratory papillomatosis using cidofovir. J Otolaryngol. 2002; 31: 333335.

35. Pransky SM, Brewster DF, Magit AE, Kearns DB. Clinical update on 10 children treated with intralesional cidofovir injections for severe recurrent respiratory papillomatosis. Arch Otolaryngol Head Neck Surg. 2000; 126: 1239-1243.

36. Mandell DL, Arjmand EM, Kay DJ, Casselbrant ML, Rosen CA. Intralesional cidofovir for pediatric recurrent respiratory papillomatosis. Arch Otolaryngol Head Neck Surg. 2004; 130: 1319-11323.

37. Durvasula VS, Richter GT. Intralesional cidofovir as adjuvant for the successful management of aggressive respiratory papillomatosis in an infant. Int $\mathrm{J}$ Pediatr Otorhinolaryngol. 2013; 77: 1912-1915.

38. Palomar-Asenjo V, Palomar-García V, Soteras-Ollé J, Ruiz-Giner A. Cidofovir activity in infantile recurrent respiratory papillomatosis. Acta Otorrinolaringol Esp. 2005; 56: 22-24.

39. Clamp PJ, Saunders MW. Systematic review of intralesional cidofovir dosing regimens in the treatment of recurrent respiratory papillomatosis. Int $\mathrm{J}$ Pediatr Otorhinolaryngol. 2013; 77 (3): 323-328.

40. Drejet S, Halum S, Brigger M, Skopelja E, Parker N. A systematic review: outcomes in adult recurrent respiratory papillomatosis treated with intralesional cidofovir or bevacizumab. Otolaryngol Head Neck Surg. 2017; 156 (3): 435-441.

41. Chadha NK, James AL. Antiviral agents for the treatment of recurrent respiratory papillomatosis: a systematic review of the English-language literature. Otolaryngol Head Neck Surg. 2007; 136 (6): 863-869.

42. Chadha NK. Intralesional cidofovir for recurrent respiratory papillomatosis: Systematic review of efficacy and safety. J Laryngol Voice. 2011; 1: 22-26. 